\title{
The Use of Mobile Contact List applications and a Context- Oriented Framework to Support their Design
}

\author{
Andreas Komninos \\ Glasgow Caledonian University \\ 70 Cowcaddens Rd. \\ Glasgow G4 OBA, UK \\ +44 1413313095
}

andreas.komninos@gcal.ac.uk

\author{
Dimitrios Liarokapis \\ Glasgow Caledonian University \\ 70 Cowcaddens Rd. \\ Glasgow G4 OBA, UK \\ +441413318251 \\ d.liarokapis@gcal.ac.uk
}

\begin{abstract}
Contact lists are one of the most frequently used applications on mobile devices. They are used not only as contact detail repositories, but also as temporary information storage for unrelated items, such as PINs or passwords. Users are reluctant to delete or remove contacts from their repositories. As such, these become increasingly large, sometimes measuring several hundred entries. In this paper we present our findings from early investigations into the use of mobile Contact Lists. We also propose a context oriented design framework to aid the speed and efficacy of the information seeking and retrieval process during the use of the Contact List application.
\end{abstract}

\section{Categories and Subject Descriptors}

H.5.2 [Information Interfaces and Presentation]: User Interfaces

\section{General Terms}

Human Factors, Design, Theory

\section{Keywords}

User studies, Mobile PIM, Context Awareness

\section{INTRODUCTION}

Although the contact list application is arguably the most frequently function of a mobile phone, surprisingly little research has been published on evaluating its usability. In 1999, Bocker and Suwita [1] examined the usability of a C10 phone found that although almost all users had no issues finding and calling a given contact from the phone book ( $94 \%$ success), this rate dropped significantly to $73 \%$ when asked to find and call the same contact from a call list. Clockar et al. also investigated the usability of several mobile phone models and found that while users typically had almost no problem calling a contact from the phonebook, there were more problems when asked to check their missed call list [2]. These findings were supported by our initial conversations with users, in which they indicated that in order to

Copyright is held by the author/owner(s).

MobileHCI'09, September 15 - 18, 2009, Bonn, Germany.

ACM 978-1-60558-281-8. avoid searching for a contact to call or text, they would resort to looking in the call list first, particularly if it was someone they remembered having talked to recently. However, as shown by [1, 2], this might not always be the best strategy to adopt in terms of effectiveness. Improving the contact list using context awareness was examined in a key paper by Oulasvirta et al. [3]. Perhaps the most significant finding therein was the fact that ultimately, in situations where social factors are likely to be key to the adoption of an application, context awareness should be used to present information to the user while leaving ultimate control over the course of action to them, rather than automating it fully. Jung et al. [4] investigated the improvement of a mobile contact list using as primary design drivers the efficiency of accessing contacts and the need to differentiate important contacts. They found that users responded very positively to being able to quickly access the top 10 contacts in terms of communication frequency, those whose birthday was soon approaching and additionally those contacts who were recently added as three special category views that helped differentiate potentially important contacts from the rest of the repository. The authors did not report any experiments in mixing the two categories in a single view and while their findings seem to align with our hypothesis for the need for differentiation, top 10 seems a rather convenient and ad-hoc number to use. Their implementation does not allow ultimate control to users, with regard to who goes into a top $\mathrm{N}$ list, fully automating this process for the users.

Given the small body of literature on mobile contact applications, perhaps the Personal Information Management community has findings to offer, which might be applicable to the design of mobile PIM tools. From the body of literature available, the work of Whittaker et al.[5] stands out as fundamentally important for the purposes of this paper. Their hypotheses that users would interact with them more frequently and that they would send and receive communication more frequently than from non-important contacts were verified to be true in field and lab experiments. Equally important was the finding that recent communication was very much more likely to have come from Important contacts.

\section{CONTEXT-ORIENTED DESIGN FOR MOBILE CONTACT APPLICATIONS}

Based on existing literature and anecdotal evidence from discussions with mobile users, we became interested in improving mobile contact lists to offer easier access to important contacts. We hypothesised that the criteria for contact importance cannot be not static; after all, users' lives and priorities change continuously during the day and a truly context-aware device 
should continuously adapt to these changing circumstances. We hypothesised that important contextual cues that relate to the importance of mobile contacts might be the following:

C1: Frequency and recency of use: Could it be proven true that this criterion, as supported by [10] and partially by [9] applies to mobile as well as email contacts?

C2: Location and temporal context: Could, location and temporal context inform the dynamics of contact list displays (some contacts are more likely to be called at different times of the day or different locations, e.g. work)?

C3: Task and activity context: Could the contact list interface with a user's calendar and try to guess their upcoming activities, who they're going to meet, and prepare itself accordingly to support the user in the likely event they need to call that person? If so, how long before or after a scheduled activity does a contact remain important?

C4: Personal preference: Would the users feel comfortable with a fully automated system making promotion choices for them, or does some form of control (even if it doesn't result in optimal performance) afford the contact list application greater satisfaction in its use?

However we also need to ask further, what is the relationship between the aforementioned context criteria (Q1)? Is one more important over the other, or does their weight also vary depending on additional types of context? Furthermore, assuming that contact importance is determinable, how does this information get relayed to the user through the contact list UI (Q2)?

\section{MOBILE CONTACTS USAGE}

We began our investigation in examining $\mathrm{C} 1$, by conducting a survey of the usage of contacts in mobile contact lists. We wrote a mobile application that uses the J2ME JSR-75 PIM API to export a copy of the contact lists of 28 subjects to a text file (26 male, 2 female, aged between 18-31 years, all students but from very diverse disciplines). In total, the participants' contact lists contained 3004 entries. We found that on average, each contact lists contained 107 entries (av. $=107.57$, stdev. $=81.46$, min. $=16$, $\max =372$ ), which shows that searching and filtering contacts has to be made on considerable corpus. This fact explains why users mentioned frequently resorting to their call lists and using special characters to "promote" contacts.

We formatted the contact lists into spreadsheets and sent these back to the participants over email, asking them to tell us for every contact therein, how long had it been since they last used that particular contact. To facilitate their input, the spreadsheet columns could only be populated with pre-determined answers. We additionally asked the participants to subjectively evaluate the importance of each of their contacts and indicate those who they perceived as very important (not only in terms of frequency of use, but also importance due to emotional, work or other reasons). Unfortunately not all participants were kind enough to return their annotated contact lists, and as such we received 14 (13 male, 1 female) responses to our request for data. We found that $31 \%$ of contacts were used in the last month and $40 \%$ in the last two. This percentage for the periods of 1-2, 2-3 and 3-6 months, totals to just $22 \%$ of contacts having been used between the last 1-6 months. Finally, it was striking to see that $46 \%$ of the contacts haven't been used for at least 6 months or were never used. The design implications from these findings are obvious - it appears that just under half of the contacts objectively don't seem to be of much use to the subjects in our experiment.

As mentioned previously, we asked our participants to indicate how many contacts they thought of as important, regardless of frequency of use. Of the 14 respondents, 9 ( 8 male, 1 female) provided this information. We found that $43 \%$ of important contacts were called weekly and a further $37 \%$ within the last month. The remaining categories have entries ranging between 1$8 \%$. These findings seem to confirm that frequency of use is a strong indicator of importance, although we cannot ignore the fact that entries exist across all frequency categories, indicating that C4 is likely an important design influence. Finally we examined the relationship between the size of a contact list and the number of important contacts contained therein. We found that the number of important contacts remained relatively steady despite the increase in size of the contact lists (Fig. 2). A contact list UI should, as such, be able to provide quick access to approximately 20 important contacts.

\section{CONCLUSIONS}

We presented a research framework comprising of the investigation of 4 context criteria for the design of adaptive mobile contact list applications. We present findings that support the importance of criterion C1 (frequency \& recency of use) in the design of mobile contact list UIs. We also found that user preference for importance (C4) should also be catered for in an adaptive UI for mobile contact lists. To evaluate the relationship between criteria, we have are currently testing an adaptive contact list UI, informed by the design principles supported by C1 \& C4. Six independent HCI experts have evaluated a range of 14 paper prototype designs and we have implemented a functional prototype, which is undergoing evaluation trials.

\section{REFERENCES}

[1] Böcker, M., Suwita, A. 1999. Evaluating the Siemens C10 mobile phone - Beyond “Quick and Dirty” Usability TestingProceedings of HFT’99, May 1999, Copenhagen, Denmark.

[2] Clockar, T., Carr, D., Hedman, A., Johansson, T., Bengtsson, F., 2003. Usability of Mobile Phones.In Proceeding of the 19th International Symposium on Human Factors in Telecommunications, Berlin, Germany, 197-204.

[3] Oulasvirta, A., Raento, M., Tiita, S. 2005. ContextContacts: Re-Designing Smartphone's Contact Book to Support Mobile Awareness and Collaboration. In Proc. MobileHCI’05, ACM Press (2005), pp. 167-174.

[4] Jung, Y., Anttila, A., Blom, J. 2008. Designing for the Evolution of Mobile Contacts Applications. In Proc. MobileHCI'08, ACM Press (2008), pp. 449-452.

[5] Whittaker, S., Jones, Q., Terveen, L., 2002. Contact Management: Identifying Contacts to Support Long-Term Communication. In Proceedings of CSCW 2002 (New Orleans, LA November 16-20, 2002), 216-225 